"The first example of isomeric solid amide and its enol"

Ahmad Basheer and Zvi Rappoport

Supporting information

Table of contents

Table of contents 1

Synthesis of 8

Table S1 4

Table S2 5

Table S3 6

${ }^{13} \mathrm{C}$ NMR spectra of 7 in DMSO- $d_{6}$

${ }^{1} \mathrm{H}$ NMR spectra of a $\mathbf{8} / \mathbf{9}$ mixture in $\mathrm{CDCl}_{3} \quad 8$

Expansion of the Me signals in the ${ }^{1} \mathrm{H}$ NMR spectrum of $\mathbf{8} / \mathbf{9}$ mixture in $\mathrm{CDCl}_{3}$

The $\mathrm{NH}$ signal of a $\mathbf{8 / 9}$ mixture in $\mathrm{CDCl}_{3}$

${ }^{13} \mathrm{C}$ NMR signal of a $\mathbf{8} / \mathbf{9}$ mixture in $\mathrm{CDCl}_{3}$

${ }^{13} \mathrm{C}$ NMR $-{ }^{1} \mathrm{H}$ coupled spectra of the Me signal of a $\mathbf{8} / \mathbf{9}$ mixture in $\mathrm{CDCl}_{3}$

cif file of 8

cif file of $\mathbf{8}$

cif file of 9 


\section{Synthesis of $\mathbf{8}$}

Reaction of N-methylcyanoacetamide 5 with Methyl Isothiocyanate 6. To a suspension of sodium $(0.24 \mathrm{~g}, 10.5 \mathrm{mmol})$ in dry THF $(50 \mathrm{~mL})$ under nitrogen was added N-methylcyanoacetamide $5(0.99 \mathrm{~g}, 10 \mathrm{mmol})$. The mixture was stirred overnight and a white precipitate was formed. A solution of methyl isothiocyanate 6 $(073 \mathrm{~g}, 10 \mathrm{mmol})$ in dry THF $(10 \mathrm{~mL})$ was added dropwise during $30 \mathrm{~min}$. The mixture was stirred at room temperature for 4 hrs during which most of the precipitate was dissolved, and then was refluxed for $2 \mathrm{hrs,}$ giving a yellow precipitate. The mixture was cooled to room temperature and the precipitate was filtered and washed with dry ether $(50 \mathrm{~mL})$ to give the sodium salt 7.

${ }^{1} \mathrm{H}$ NMR (DMSO-d $\left.6,298 \mathrm{~K}\right) \delta: 2.54(3 \mathrm{H}, \mathrm{d}, \mathrm{J}=4.2 \mathrm{~Hz}, \mathrm{CSNMe}), 2.89$ (3H, d, J $=4.4 \mathrm{~Hz}, \mathrm{CONMe}), 5.80(1 \mathrm{H}, \mathrm{s}, \mathrm{CSN} H), 10.63(1 \mathrm{H}, \mathrm{s}, \mathrm{CON} H) ;{ }^{13} \mathrm{C} \mathrm{NMR}\left(\mathrm{DMSO}-\mathrm{d}_{6}\right.$,

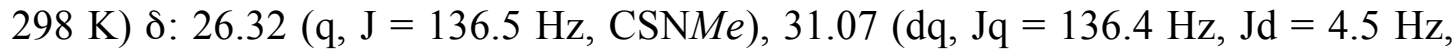
CSNMe), $72.66\left(\mathrm{~s}, \mathrm{C}^{-}\right), 126.77(\mathrm{~s}, \mathrm{CN}), 170.18(\mathrm{~s}, \mathrm{C}=\mathrm{O}), 189.49(\mathrm{~s}, \mathrm{C}=\mathrm{S})$.

7 was dissolved in DMF (5 mL) and the solution was added dropwise to an icecold solution of $2 \mathrm{~N} \mathrm{HCl}(50 \mathrm{~mL})$. The white solid formed was filtered and washed with cold water $(100 \mathrm{~mL})$ and dried at $\mathrm{rt}$ giving $1.57 \mathrm{~g}(92 \%)$ of the pure product.

Anal. Calcd for $\mathrm{C}_{6} \mathrm{H}_{9} \mathrm{~N}_{3} \mathrm{OS}$ : C, 42.11; H, 5.26; N, 24.56. Found: C, 42.39; H, $5.26 ; \mathrm{N}, 24.46 \%$.

Suitable crystals of $\mathbf{9}, \mathrm{mp} 137{ }^{\circ} \mathrm{C}$ for X-ray diffraction were obtained by dissolving the product in $\mathrm{CDCl}_{3}$, and keeping the solution at $\mathrm{rt}$ for $24 \mathrm{hrs}$. Crystallization from $\mathrm{CD}_{3} \mathrm{CN}$ for $48 \mathrm{hrs}$ gave 8, mp 139-40 ${ }^{\circ} \mathrm{C}$.

${ }^{1} \mathrm{H}$ NMR $\left(\mathrm{CDCl}_{3} 298 \mathrm{~K}\right)$ displayed signals for 22:71:7 8:9:10 mixture. $\delta(\mathbf{8})$ : $2.86(\mathrm{~d}, \mathrm{~J}=4.8 \mathrm{~Hz}, \mathrm{CSNMe}), 3.17(\mathrm{~d}, \mathrm{~J}=4.7 \mathrm{~Hz}, \mathrm{CONMe}), 4.88(\mathrm{~s}, \mathrm{CH}), 7.06(\mathrm{~s}$, 
CSNH), 9.17 (s, CONH). $\delta(9): 2.96(\mathrm{~d}, \mathrm{~J}=4.7 \mathrm{~Hz}, \mathrm{CSN} M e), 3.13$ (d, J = 4.6 Hz, CONMe), 6.13 (s, CSNH), 6.90 (s, CONH) 16.59 (s, OH). $\delta(\mathbf{1 0}): 2.80$ (d, J = $4.4 \mathrm{~Hz}$, CSNMe), 3.107 (d, J = 4.7 Hz, CONMe), 4.55 (s, SH), 5.82 (s, CONH), 11.02 (s, $\mathrm{CSN} H)$.

Additional ${ }^{1} \mathrm{H}$ and ${ }^{13} \mathrm{C}$ NMR data in different solvents are given in Tables S1 and $\mathrm{S} 2$ respectively. 


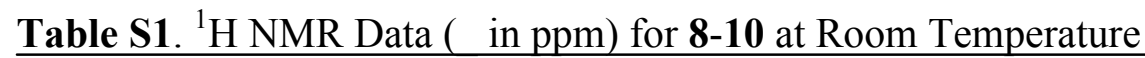

\begin{tabular}{lcccccc} 
Solvent & Species & CSNMe $^{\mathrm{a}}$ & CONMe $^{\mathrm{a}}$ & $\mathrm{CSNH}^{\mathrm{b}}$ & $\mathrm{CSN} H^{\mathrm{b}}$ & $\mathrm{CH}, \mathrm{OH}$, or SH \\
\hline $\mathrm{CDCl}_{3}$ & $\mathbf{8}$ & 2.86 & 3.17 & 7.06 & 9.17 & 4.88 \\
& $\mathbf{9}$ & 2.96 & 3.13 & 6.13 & 6.90 & 16.59 \\
& $\mathbf{1 0}$ & 2.80 & 3.07 & 11.02 & 5.82 & 4.55 \\
$\mathrm{C}_{6} \mathrm{D}_{6}$ & $\mathbf{8}$ & 2.08 & $2.47^{\mathrm{c}}$ & 5.78 & 8.35 & 4.17 \\
& $\mathbf{9}$ & 1.99 & 2.47 & 5.24 & 6.40 & 17.20 \\
& $\mathbf{1 0}$ & 1.81 & 2.18 & 11.04 & 5.51 & $\mathrm{~d}$ \\
$\mathrm{THF}-\mathrm{d}_{8}$ & $\mathbf{8}$ & 2.73 & 3.07 & 7.74 & 9.62 & 4.85 \\
& $\mathbf{9}$ & 2.87 & 3.04 & 7.30 & 7.74 & 16.74 \\
$\mathrm{CD}_{3} \mathrm{CN}$ & $\mathbf{8}$ & 2.74 & 3.08 & 6.94 & 8.95 & 4.88 \\
& $\mathbf{9}$ & 2.86 & 3.03 & 6.55 & 7.26 & 16.60 \\
$\mathrm{DMSO}-\mathrm{d}_{6}$ & $\mathbf{8}$ & 2.64 & 2.99 & 8.12 & 10.34 & 5.11 \\
\hline
\end{tabular}

${ }^{\mathrm{a}}$ Doublet, $\mathrm{J}=4.5-4.8 \mathrm{~Hz} .{ }^{\mathrm{b}}$ Broad singlet. ${ }^{\mathrm{c}}$ Overlaps the signal of 9. ${ }^{\mathrm{d}}$ Not observed 
Table S2. ${ }^{13}$ C NMR Data (_ in ppm) for 8-10 at Room Temperature (based on C-H coupled spectrum)

\begin{tabular}{|c|c|c|c|c|c|c|}
\hline Solvent & Species & $\mathrm{CH}$ or $\mathrm{C}_{-}$ & $\begin{array}{l}\text { CSNMe, } \\
\text { CONMe }\end{array}$ & $\mathrm{CN}$ & $\begin{array}{l}\mathrm{C}=\mathrm{O} \text { or } \\
\mathrm{C}=\mathrm{S}\end{array}$ & $\begin{array}{l}\mathrm{C}_{-} \text {or } \\
\mathrm{C}=\mathrm{O}\end{array}$ \\
\hline \multirow[t]{3}{*}{$\mathrm{CDCl}_{3}$} & 8 & $\begin{array}{l}54.15(\mathrm{~d}, \\
\mathrm{J}=139.9 \\
\mathrm{~Hz})\end{array}$ & $27.27,33.70$ & $\begin{array}{l}114.37(\mathrm{~d}, \\
\mathrm{J}=10.3 \\
\mathrm{~Hz})\end{array}$ & $187.67(\mathrm{~m})$ & $161.61(\mathrm{~m})$ \\
\hline & 9 & $66.03(\mathrm{~s})$ & $27.34,30.84$ & $118.58(\mathrm{~s})$ & $\begin{array}{l}187.36(\mathrm{q}, \\
\mathrm{J}=4.5 \mathrm{~Hz})\end{array}$ & $171.63(\mathrm{~m})$ \\
\hline & 10 & $70.20(\mathrm{~s})$ & $26.05,32.13$ & $121.45(\mathrm{~s})$ & $167.77(\mathrm{~s})$ & $173.26(\mathrm{~s})$ \\
\hline \multirow[t]{2}{*}{$\mathrm{C}_{6} \mathrm{D}_{6}{ }^{\mathrm{b}}$} & 8 & $\begin{array}{l}53.88(\mathrm{~d}, \\
\mathrm{J}=140.7 \\
\mathrm{~Hz})\end{array}$ & 32.66 & $\begin{array}{l}116.72(\mathrm{~d}, \\
\mathrm{J}=9.9 \\
\mathrm{~Hz})\end{array}$ & $189.16(\mathrm{~m})$ & $161.60(\mathrm{~m})$ \\
\hline & 9 & 66.39(s) & $26.29,30.05$ & $118.06(\mathrm{~s})$ & $187.69(\mathrm{~s})$ & $171.60(\mathrm{~m})$ \\
\hline \multirow[t]{2}{*}{ THF-d 8} & 8 & $\begin{array}{l}53.74(\mathrm{~d}, \\
\mathrm{J}=140.8 \\
\mathrm{~Hz})\end{array}$ & $26.08,32.75$ & $\begin{array}{l}114.28(\mathrm{~d}, \\
\mathrm{J}=9.8 \\
\mathrm{~Hz})\end{array}$ & $188.55(\mathrm{~m})$ & $161.49(\mathrm{~m})$ \\
\hline & 9 & $65.76(\mathrm{~s})$ & $26.44,30.11$ & $116.86(\mathrm{~s})$ & $\begin{array}{l}187.26(\mathrm{q}, \\
\mathrm{J}=4.7 \mathrm{~Hz}\end{array}$ & $171.44(\mathrm{~m})$ \\
\hline \multirow[t]{2}{*}{$\mathrm{CD}_{3} \mathrm{CN}$} & 8 & $\begin{array}{l}53.70(\mathrm{~d}, \\
\mathrm{J}=139.2 \\
\mathrm{~Hz})\end{array}$ & $26.38,33.02$ & $\begin{array}{l}114.87(\mathrm{~d}, \\
\mathrm{J}=10.4 \\
\mathrm{~Hz})\end{array}$ & $188.93(\mathrm{~s})$ & $161.60(\mathrm{~m})$ \\
\hline & 9 & $65.77(\mathrm{~s})$ & $26.63,30.23$ & $117.23(\mathrm{~s})$ & 186.97(s) & $171.45(\mathrm{~s})$ \\
\hline $\mathrm{DMSO}_{-} \mathrm{d}_{6}$ & 8 & $\begin{array}{l}53.36(\mathrm{~d}, \\
\mathrm{J}=137.6 \\
\mathrm{~Hz})\end{array}$ & $27.15,33.77$ & $\begin{array}{l}115.88(d, \\
J=9.7 \\
H z)\end{array}$ & $189.06(\mathrm{~s})$ & $161.63(\mathrm{~s})$ \\
\hline
\end{tabular}

${ }^{\mathrm{a}}$ Quartet, $\mathrm{J}=138.1-141.2 \mathrm{~Hz} .{ }^{\mathrm{b}}$ The signals of $\mathbf{1 0}$ were not observed in $\mathrm{C}_{6} \mathrm{D}_{6}$. 
Table S3. ${ }^{1} \mathrm{H}$ NMR data (_ in ppm) and Integrals for 8-10 in $\mathrm{CDCl}_{3}$

\begin{tabular}{ccccccc} 
Species & $\begin{array}{c}\text { Shift and } \\
\text { integrals }\end{array}$ & CSNMe & CONMe & CSNH & CSNH & $\begin{array}{c}\text { CH, OH or } \\
\text { SH }\end{array}$ \\
\hline $\mathbf{8}$ & shift & 2.86 & 3.17 & 7.06 & 9.17 & 4.88 \\
& integral & 0.96 & 0.95 & 0.31 & 0.29 & 0.30 \\
$\mathbf{9}$ & shift & 2.96 & 3.13 & 6.13 & 6.90 & 16.59 \\
& integral & 3.00 & 2.95 & 0.99 & 0.98 & 1.01 \\
$\mathbf{1 0}$ & shift & 2.80 & 3.07 & 11.02 & 5.82 & 4.55 \\
& integral & 0.27 & 0.27 & 0.08 & 0.08 & 0.07 \\
\hline
\end{tabular}




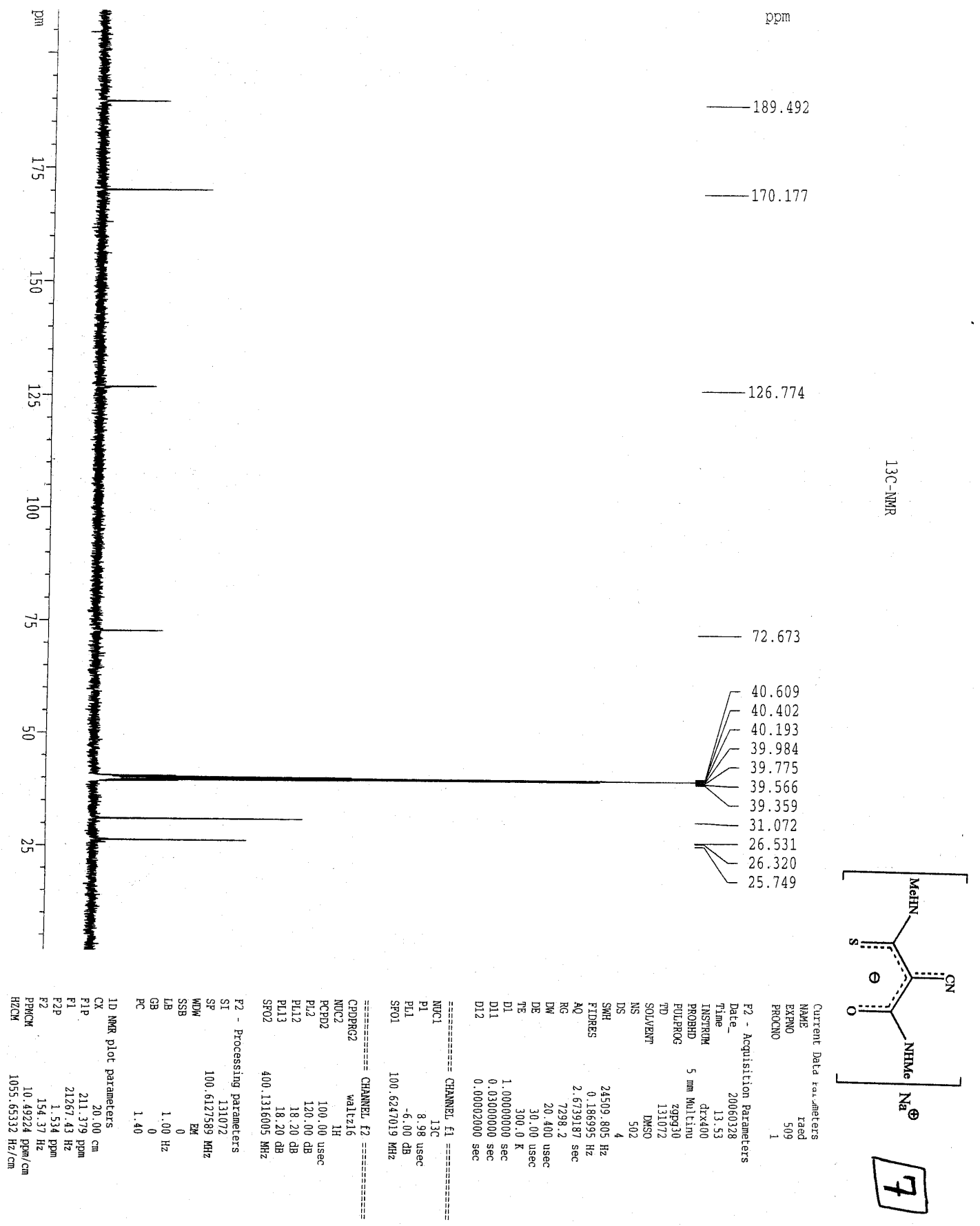




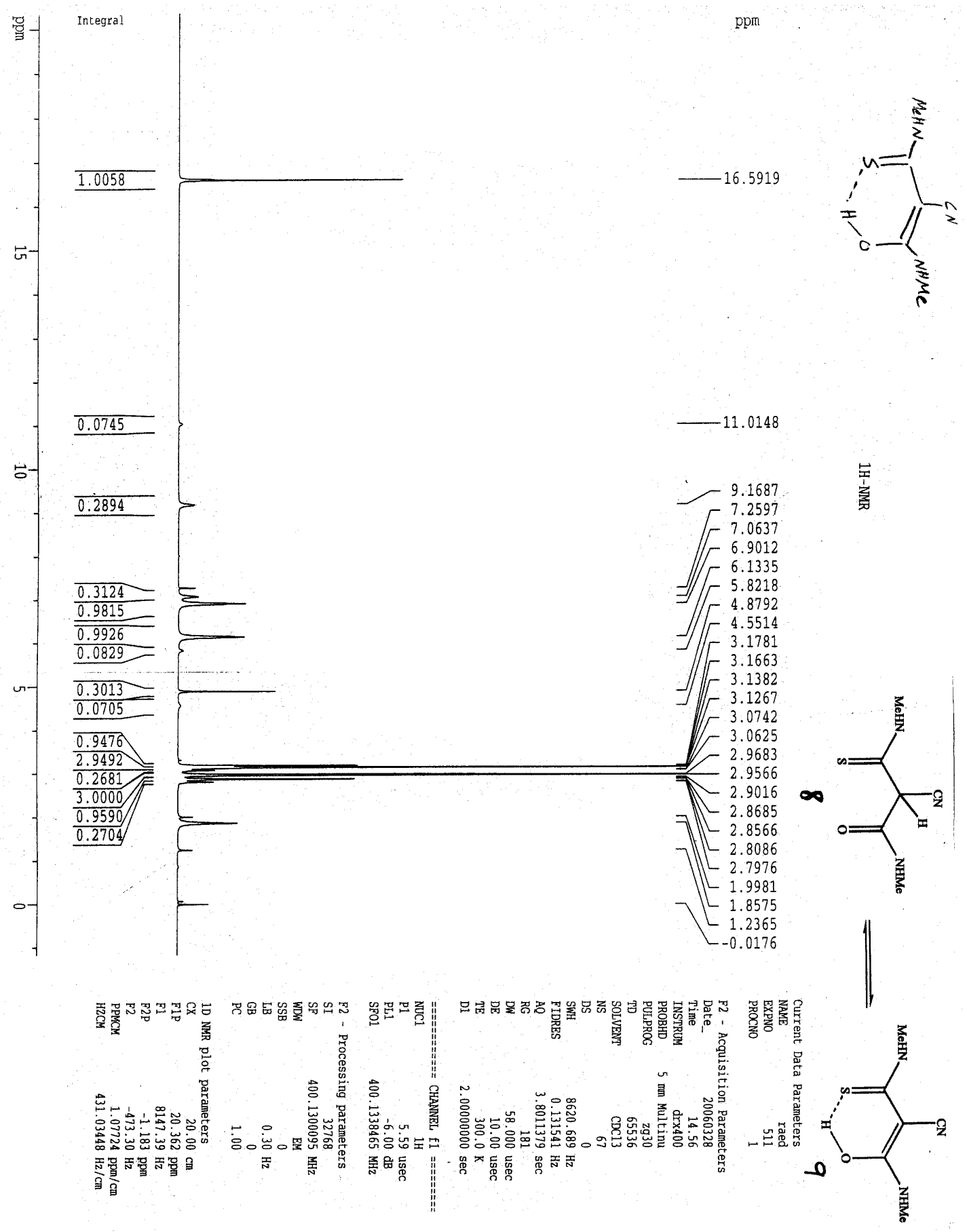




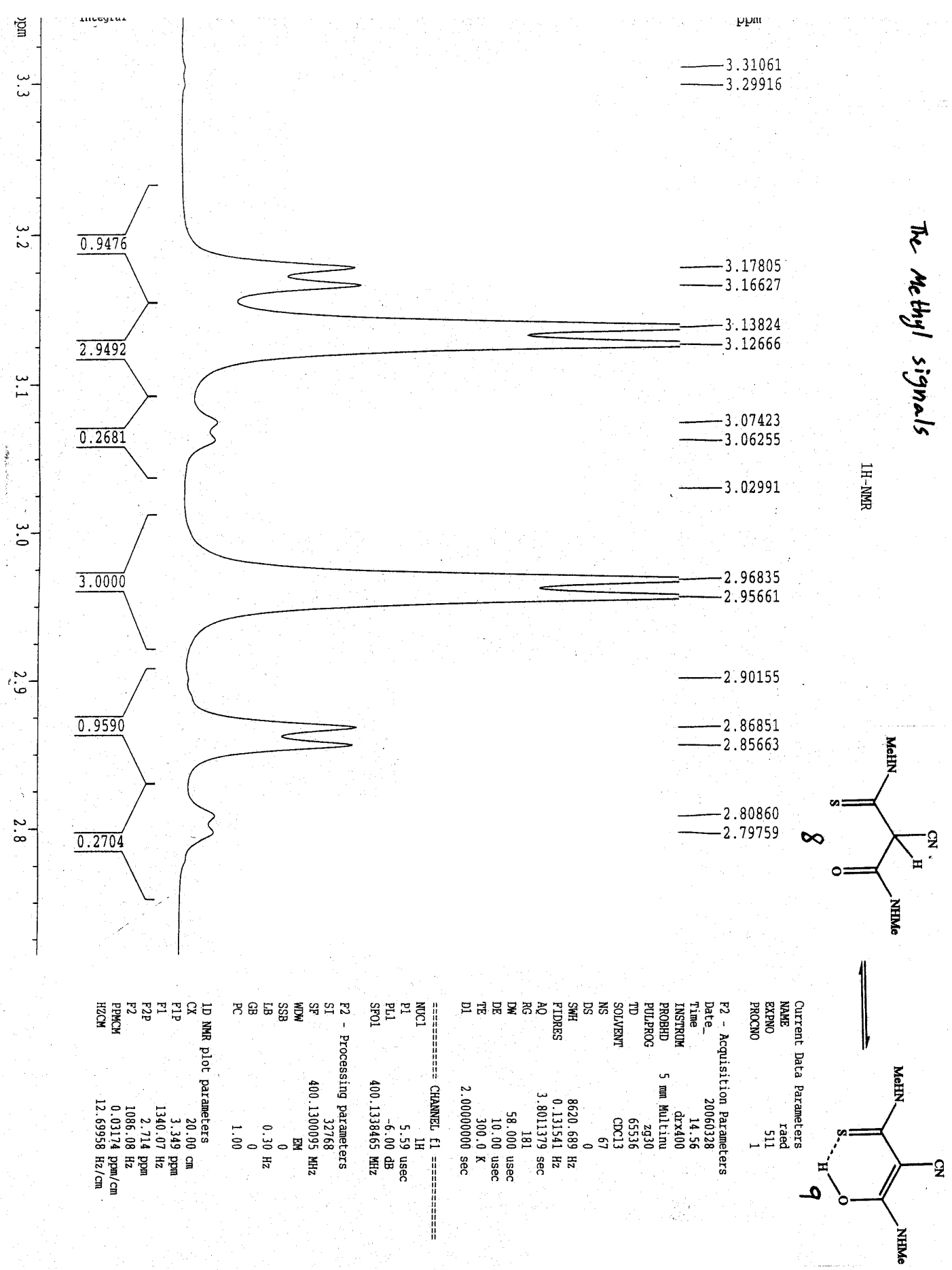




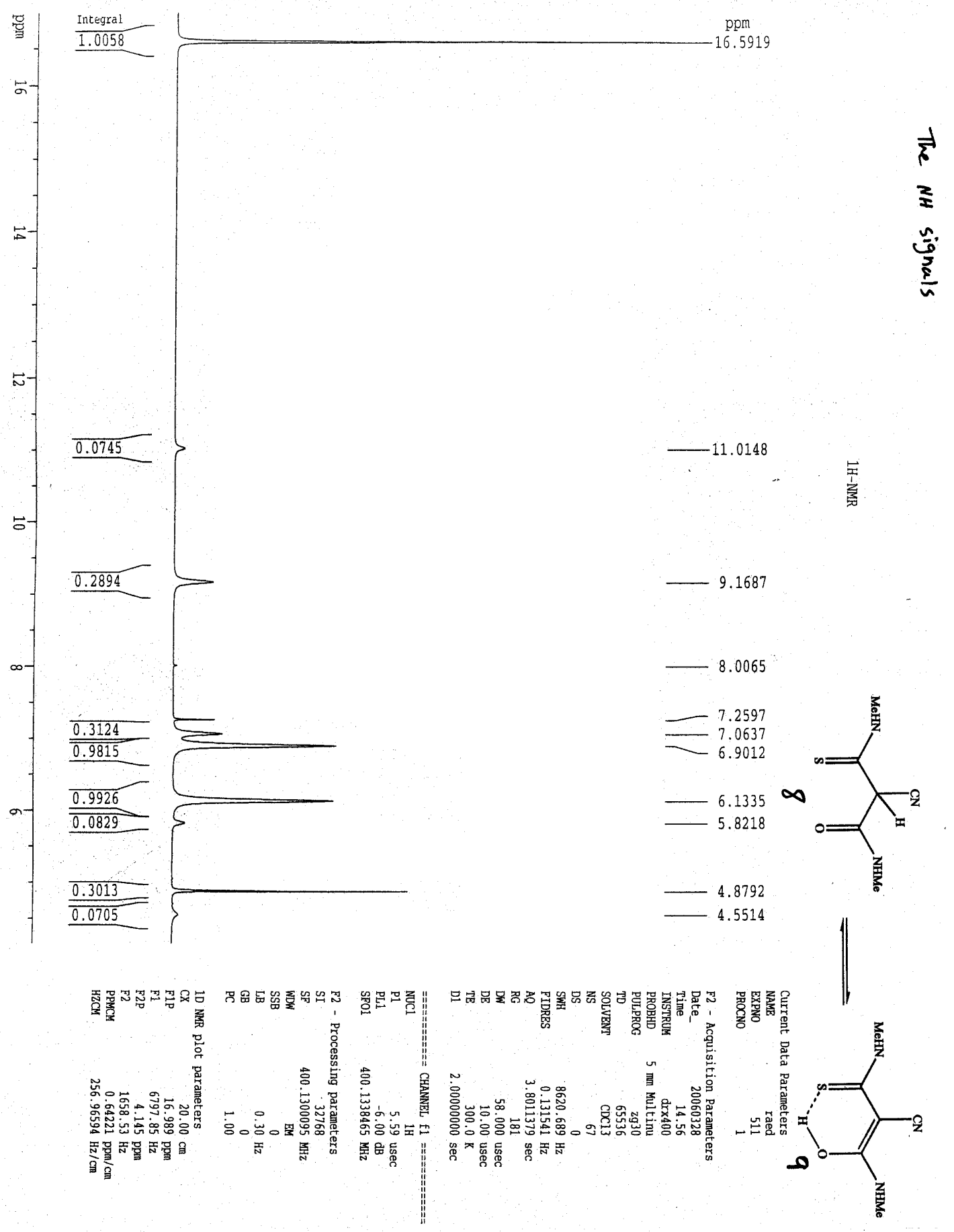




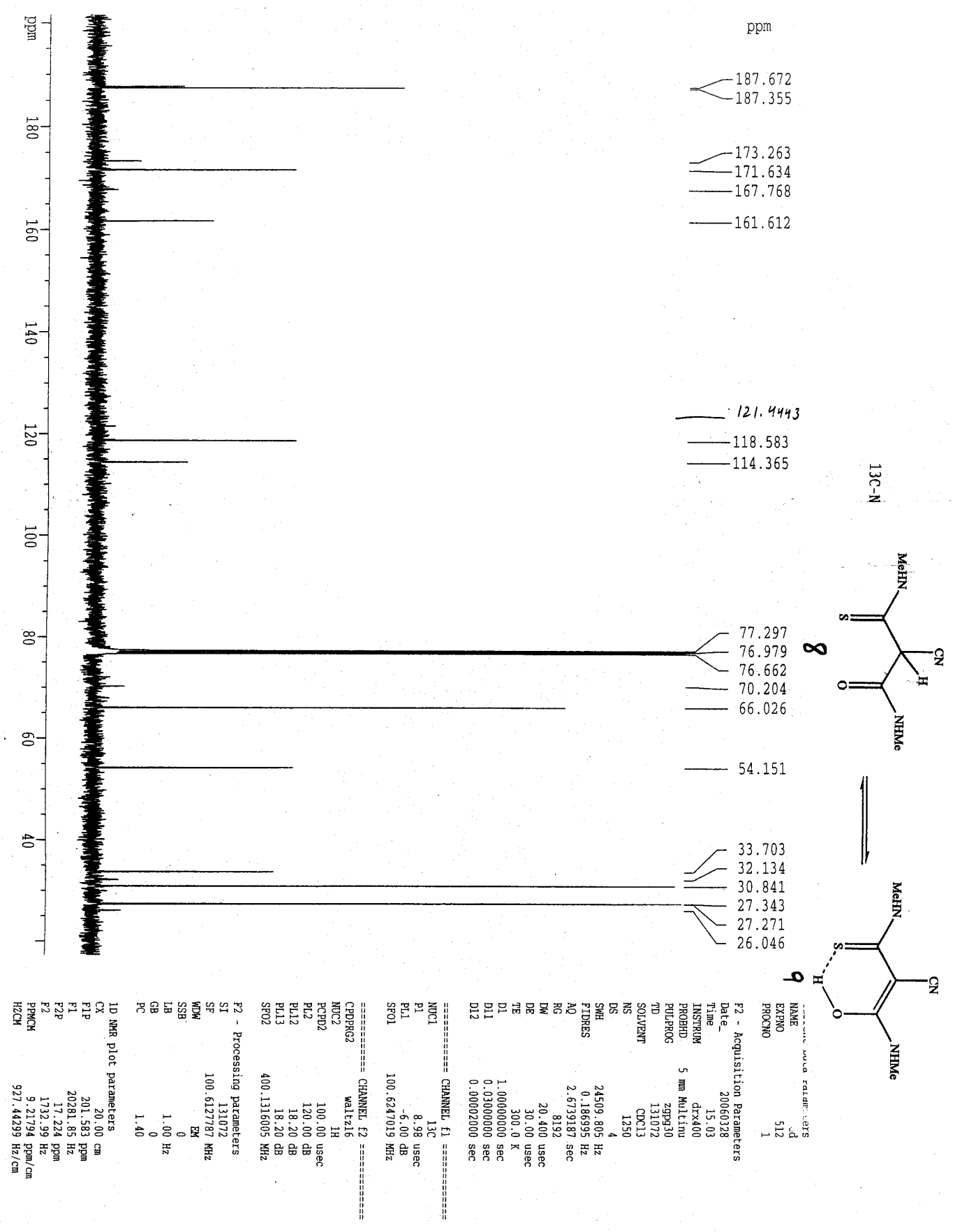



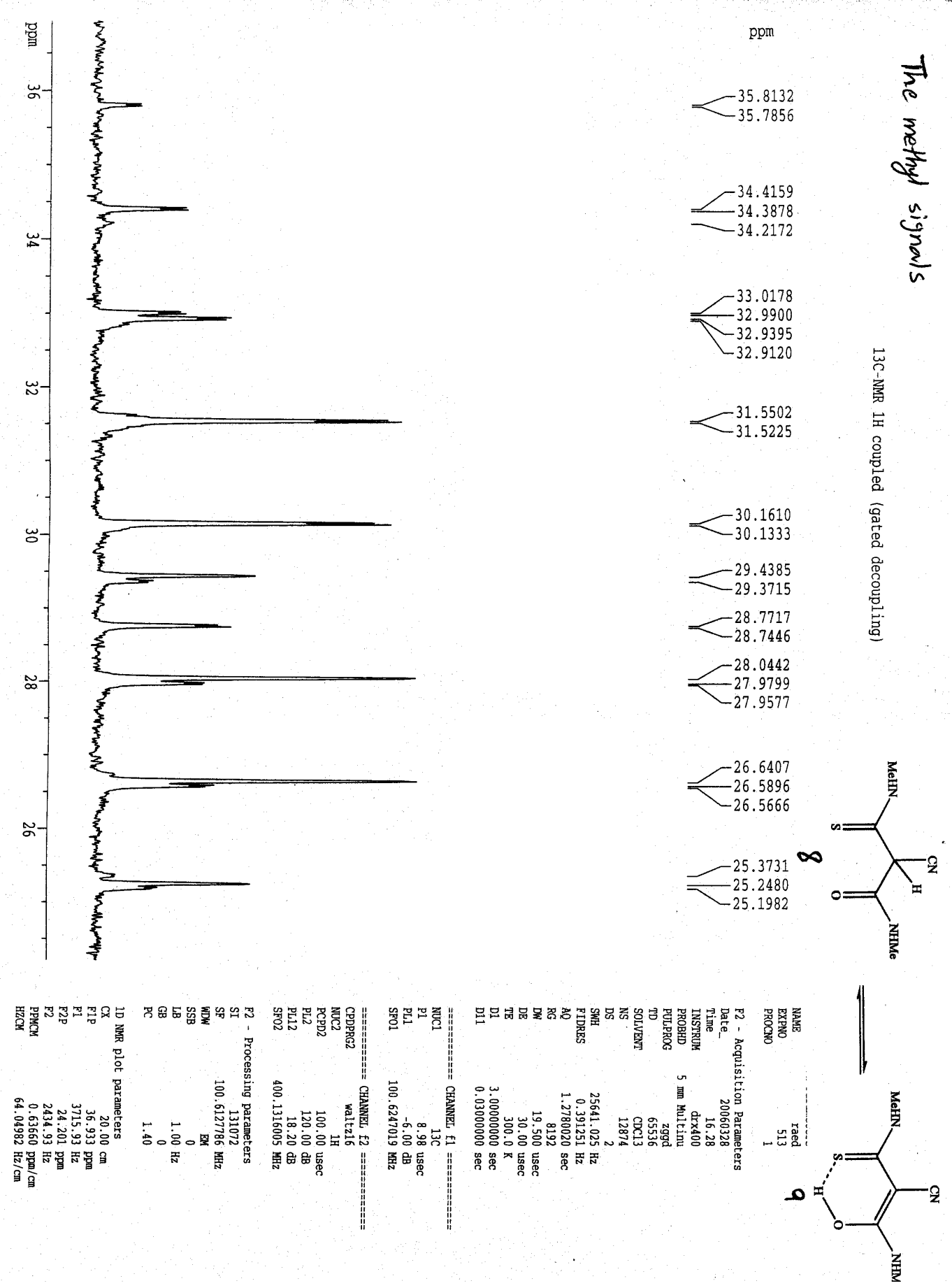